\title{
Flashing light in sponges through their siliceous fiber network: A new strategy of "neuronal transmission" in animals
}

\author{
WANG XiaoHong ${ }^{1,2 *}$, FAN XingTao ${ }^{1}$, SCHRÖDER Heinz $C^{2} \&$ MÜLLER Werner E G ${ }^{2 *}$ \\ ${ }^{1}$ National Research Center for Geoanalysis, Chinese Academy of Geological Sciences, Beijing 100037, China; \\ ${ }^{2}$ ERC Advanced Grant Research Group at the Institute for Physiological Chemistry, University Medical Center of the Johannes Gutenberg \\ University, Mainz D-55099, Germany
}

Received November 7, 2011; accepted April 20, 2012; published online July 11, 2012

\begin{abstract}
Sponges (phylum Porifera) represent a successful animal taxon that evolved prior to the Ediacaran-Cambrian boundary (542 million years ago). They have developed an almost complete array of cell- and tissue-based interaction systems necessary for the establishment of a functional, multicellular body. However, a network of neurons, one cell/tissue-communication system is missing in sponges. This fact is puzzling and enigmatic, because these animals possess receptors known to be involved in the nervous system in evolutionary younger animal phyla. As an example, the metabotropic glutamate/GABA-like receptor has been identified and cloned by us. Recently, we have identified a novel light transmission/light responsive system in sponges that is based on their skeletal elements, the siliceous glass fibers, termed spicules. Two classes of sponges, the Hexactinellida and the Demospongiae, possess a siliceous skeleton that is composed of spicules. Studying the large spicules from hexactinellid sponges $(>5 \mathrm{~cm})$ revealed that these spicules are effective light-collecting optical fibers. Now we can report that the demosponge, Suberites domuncula, has a biosensor system consisting of the (organic) light producing luciferase and the (inorganic) light transducing silica spicules. The light transmission features of these smaller spicules $(200 \mu \mathrm{m})$ has been demonstrated and the ability of the sponge tissue to generate light had been proven. Screening for a luciferase gene in $S$. domuncula was successful. In the next step, we searched for a protein potentially involved in light reception. Such a protein was identified, cloned and recombinantly expressed from S. domuncula. The protein sequence displays two domains characteristic of a cryptochrome, the N-terminal photolyase-related region and the C-terminal FAD-binding domain. The experimental data indicate that sponges may employ a network of luciferase-like proteins, a spicular system and a cryptochrome as the light source, optical waveguide and photosensor, respectively. Finally, we have identified a potential transcription factor involved in the control of the expression of luciferase and cryptochrome, a SOX-related protein. We assume that a flashing light signaling circuit exists, which may control the retinoic acid-induced differentiation of stem cells into pulsating and contracting sponge cells, and into pinacocytes and myocytes. Such a "nervous"-like signal transduction system has not been previously described.
\end{abstract}

sponges, Suberites domuncula, “nervous”-like signal system, light transmission, siliceous spicules, luciferase, cryptochrome

Citation: Wang X H, Fan X T, Schröder H C, et al. Flashing light in sponges through their siliceous fiber network: A new strategy of "neuronal transmission" in animals. Chin Sci Bull, 2012, 57: 3300-3311, doi: 10.1007/s11434-012-5241-9

In living organisms 4 major groups of biominerals exist: (1) iron compounds which are primarily restricted to prokaryotes; (2) calcium phosphates found in metazoans; (3) calcium carbonates used by prokaryotes, protozoans, plants, fungi and metazoans; and (4) amorphous silica present in sponges and diatoms. It is surprising that the occurrence of

*Corresponding authors (email: wxh0408@hotmail.com; wmueller@uni-mainz.de) silica as a major skeletal element is restricted to particular unicellular organisms and to sponges (Demospongiae and Hexactinellida). Since the transition from Protozoa to Metazoa dates back to 600 to 1000 million years ago, it has been proposed that the oxygen level, temperature and seawater chemistry played a major role in the evolution from Protozoa to Metazoa [1,2]. In particular, it is postulated that during the period of the appearance of sponges the ocean 
was richer in sodium carbonate than in sodium chloride, and that such a "soda ocean" had probably a $\mathrm{pH}>9$. Under such conditions the concentration of silica, the dioxide form of silicon, in seawater was presumably higher than it is today.

Even though these animals, the sponges, comprise the simplest body plan [3], their biomineral structure formation is already highly complex and poorly understood. Like in triploblasts, the diploblastic Porifera skeleton formation also has a pronounced effect on morphogenesis. As an example, if animals grow under unfavorable conditions that do not allow the formation of inorganic deposits (silica or calcium biominerals), the growth of the specimens is heavily suppressed. Silica is the major constituent of sponge spicules in the classes of Demospongiae and Hexactinellida [4,5]. The spicules of these sponges are composed of hydrated, amorphous and non-crystalline silica. The secretion of spicules occurs in Demospongiae in specialized cells, the sclerocytes, in which silica is deposited around an organic filament. If the formation of siliceous spicules is inhibited, the sponge body collapses. The synthesis of spicules is a rapid process with $100 \mu \mathrm{m}$ long megascleres forming within $40 \mathrm{~h}$. Recent studies revealed that the dominant enzyme that catalyzes the formation of monomeric to polymeric silica is silicatein, an enzyme that belongs to the cathepsin subfamily [6,7]. The formation of siliceous spicules in sponges is genetically controlled, and in turn, also the processes ruling morphogenesis [8]. The skeletal framework of the sponges is highly ordered, as clearly observed in the examples of the demosponge Lubomirskia baicalensis [9] and the hexactinellid Monorhaphis chuni [5]. Most siliceous sponges are composed of longer megascleres, $>10 \mu \mathrm{m}$ in length, and shorter microscleres $(<10 \mu \mathrm{m})$.

\section{Spicule network in sponges: a unique nervous system?}

Sponges are devoid of a nervous system. We have proposed that the biological function of the siliceous spicules is to act as optical fibers that may substitute for a nerve system [10]. For our initial studies, the hexactinellid sponge species $\mathrm{Hy}$ alonema sieboldi was selected, which is characterized by extremely long spicules that form the stalk of the animals. Subsequently, the same function was also proposed for the hexactinellid $M$. chuni [11]. The first surprising result was that light transmitted through the spicules was cut-off below $600 \mathrm{~nm}$ and above $1310 \mathrm{~nm}$, in a similar manner to a combined high/low pass filter [10,11]. From comprehensive studies of luminous genera, which includes organisms that are able to emit light through their bioluminescent systems, it is known that most pelagic deep-sea organisms emit light with emission spectra maxima around $480 \mathrm{~nm}$, whereas those which are terrestrial or live in freshwater produce light with longer wavelengths. Bioluminescence, a widespread phenomenon in nature, is a process in which two molecules of an organism react by the emission of light.

\section{Light production in dark aquatic environments}

Luminous bacteria occur frequently in the aquatic/marine environment and are found as planktonic forms and in symbioses with fish, squid and other organisms [12,13] (Figure 1(a)). In a classic experiment, fish kept for 1 to $2 \mathrm{~d}$ in the open are shown to have been settled by bacteria. Colonies of luminous bacteria start to grow and can be photographed to show the production of their own light (Figure 1(a)). This visible light is made by living organisms, and hence this light quality was termed as bioluminescence. On land this phenomenon is rarely seen, with fireflies representing the best-known terrestrial example of living light. In contrast, in the ocean bioluminescence is very common in pro- and eukaryotic organisms ranging in sizes from microns to meters, and from autotrophs, herbivores to carnivores. Bioluminescence as a form of chemiluminescence is well known, where the reaction chemicals are produced by living organisms. The names assigned to these organic chemicals are luciferin for the substrate and luciferase for the enzyme. Bioluminescence light allows these organisms to passively attract or actively repel commensalic organisms. However, it has never been proposed that light is also a signal conductor within an organism. In the ocean, blue light can penetrate deepest (Figure 1(c)) and the relief area can be resolved on the basis of different intensities of a blue color. If the same area is photographed with a flash, a bright scale of colors becomes visible (Figure 1(d)).

By checking adaptation of organisms in the dark, two light qualities are distinguished: bioluminescence and biofluorescence. This can be strikingly seen in animals that live in absolute darkness, e.g., in caves. The "grottenolm", the hypogean salamander Proteus anguinus, is an example who lives in the Adriatic karst caves and is blind. However, after illumination with a daylight flash the biofluorescent signals arising from the pigment (hemoglobin) of the almost transparent reptile become apparent (Figure 1(b)). The fluorescence of animals under these conditions has no function for the behavior of the animals in the dark [14]. A different function of this bio-phenomenon can be attributed to the biofluorescence seen in other aquatic organisms. In corals this property has been attributed to a behavioral response [15]. Biofluorescence is also often seen in sponges, e.g., in the Baikalian sponge Lubomirskia baicalensis [16]. This sponge species lives in a symbiotic relationship with dinoflagellates [17], a form of interaction that allows the sponges to survive in very adverse situations under the ice cover for six months of the year. By diving down to these specimens (Figure 1(e)) it is possible to clearly observe that most of the specimens have a deep green color that reflects the richness of chlorophyll residing in the dinoflagellates on 

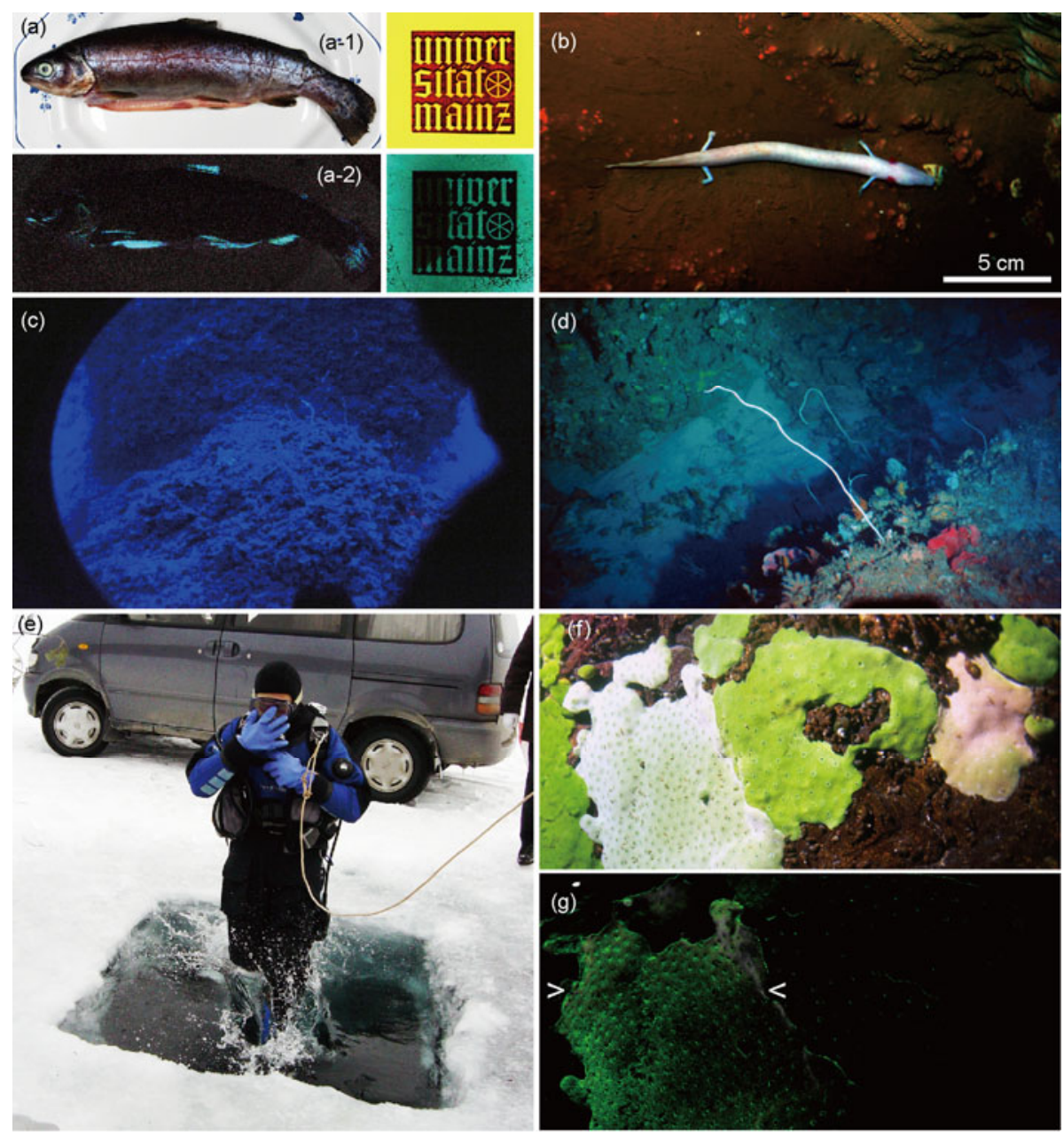

Figure 1 Bioluminescence/fluorescence in aquatic organisms. (a) Bacteria grow on a fish lying on a plate for 1-2 d; (a-1); daylight image. Frequently, luminous bacteria that have formed colonies on the surface of the fish can be photographed by their own light (a-2); dark image. In the right panel, a mask placed on top of the bacteria colony blocked bacteria-generated light (logo of the University Mainz). (b) A "grottenolm" (Proteus anguinus) photographed in a cave of the Adriatic karst. The blind animals are colored because of the hemoglobin transported through the body and is very richly accumulated in the pink external gills. (c) At a depth of $300 \mathrm{~m}$ in the tropical sea, underwater images taken with a natural light can resolve only various blue qualities. (d) In contrast, if a photo of the same area is taken using a flash the surrounding is colorful. (e) Diving to the Baikalian sponge Lubomirskia baicalensis, living under 1-2 $\mathrm{m}$ thick ice covers. (f) A photo taken with a daylight flash showing the sponges with different color variations from green to whitish. The color is dependent on the amount of chlorophyll their algal symbionts have. (g) Illumination of those specimens in the dark highlights the biofluorescence residing in the oscules of the specimens (marked: $><$ ); it is suggested that the signals emanating from those areas may be involved in a symbiotic interaction with the microorganisms.

those sponges. Illuminating them in the dark with daylight shows their green color (Figure 1(f)). However, if they are illuminated with blue light some of these specimens give a green fluorescence (Figure $1(\mathrm{~g})$ ). This color is attributed to the accumulation of chlorophyll; however, it may also have a function in attracting microorganisms especially around the oscule region, as had been proposed by Wilson and Hastings [18].

\section{Organization of the spicules within the sponge body}

The organization of the spicules within the organism is genetically controlled. The spicule-based skeleton of sponges is architecturally complex and arranged in a functionally efficient manner. As already outlined in the Challenger Report [19], two main types of spicule arrangements are realized in demosponges, the "Radiate" and the "Reticulate". The radial and also the spiral arrangement of spicules follows a genetically determined pattern (Figure 2(a)). The spiral organization is characteristic, for example, of the Tethyidae, and the radial skeleton is exemplified by the $\mathrm{Su}-$ beritidae (Figure 2(b)). The central as well as the peripheral skeleton of $S$. domuncula is composed of spicules that are oriented along the aquiferous canal system. In hexactinellids, e.g., in Euplectella aspergillum, the interaction between the spicules is even more obvious. Their spicules grow, on average, to larger sizes [20] and often fuse to form a continuous super-scaffold [21] (Figure 2(c) and (e)). The tuned interaction of the spicules becomes especially apparent from images taken inside the sponge skeleton (Figure 2(d)). From those 

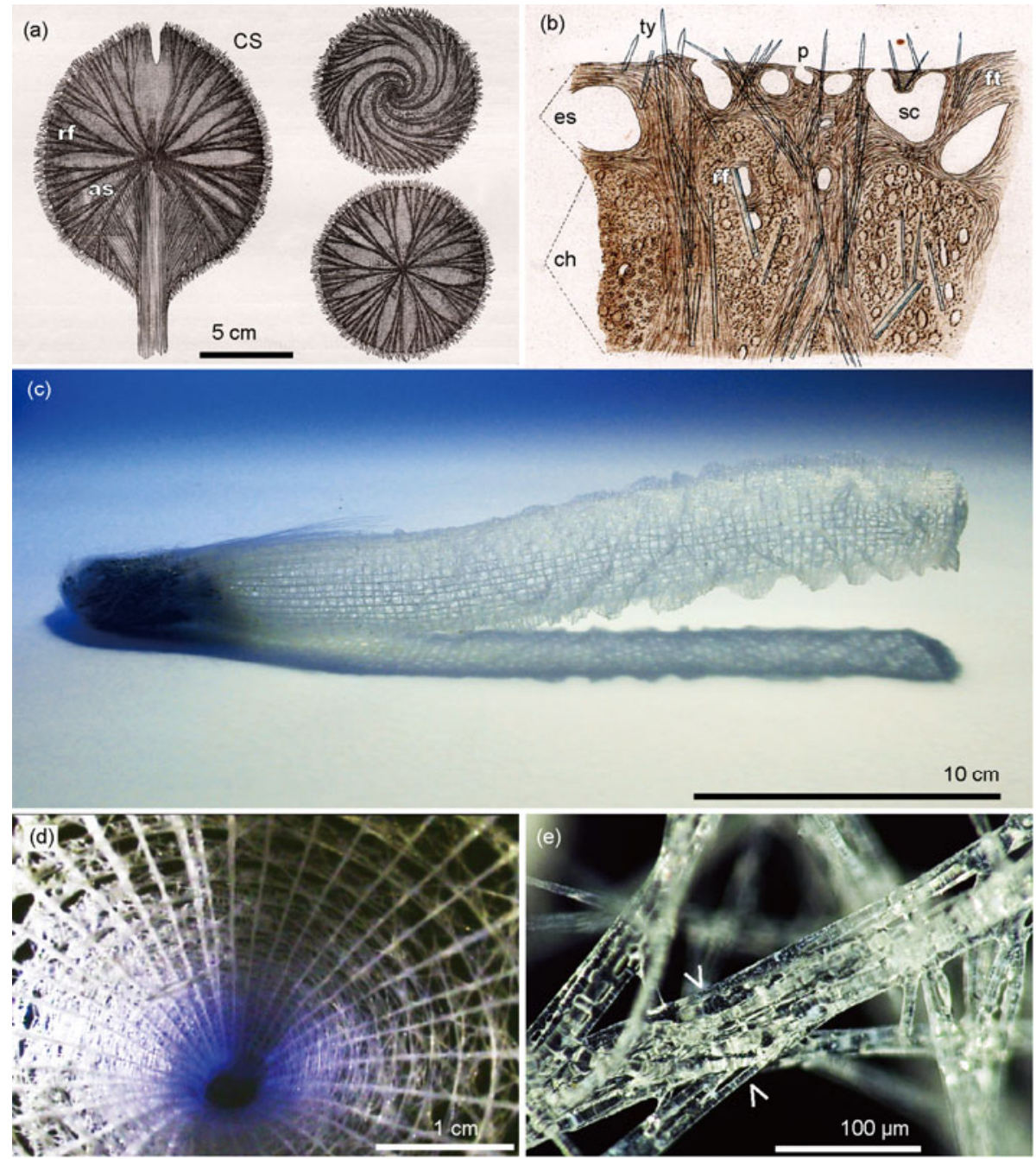

Figure 2 Spicule-interacting network. (a) Schematic representation of the skeletal organization in siliceous sponges (Demospongiae): "radiate" pattern of the spiral type, the spicules are arranged in bundles that have been termed radiating skeletal fibers (rf). Within the fibers, the tissue is organized and harbors the aquiferous canal system (as). At the surface of the animals, the radiating skeletal fibers splice into the individual cortical spicules (cs); from Ridley and Dendy [19]. (b) A cross-section through Suberites perfectus displays a more detailed description of the radial organization at the surface of this species [19]. The surface tissue is organized into the ectosome (es), which is layered on top of the choanosome (ch). The ectosome consists of brushes of nearly uniform spicules, tylostyles (ty). Individual spicules emerge from the radiating skeletal fibers (rf) and protrude into the surrounding milieu. Those spicules shelter pores (p) that function as ingestion openings for the aquiferous canal system. The pores lead to subdermal cavities (sc). The radiating skeletal fibers reinforce the fibrous tissue (ft) that is composed of individual cells embedded in a collagen network. (c)-(e) Euplectella aspergillum. The skeleton of the hexactinellid E. aspergillum is built of tightly interacting spicules. In (e) it is visible that those spicules even fuse, bio-sinter, together $(><)$.

images it is very obvious that the spicule-network renders a strong mechanical stability. Furthermore, it is suggestive that those interacting spicules may also be ideally used as a light transmission system (Figure 2(e)).

\section{Light harvesting spicules}

Based on those findings our group postulated that sponges coordinate their sensory reception systems not on the basis of a protein-controlled nervous network, but by their inorganic siliceous spicule system [10]. Subsequently, cellular and molecular biological studies were performed to substantiate this function. S. domuncula has been used as the species for these studies (Figure 3(a)). The red sponges display on their surface protruding spicules, most of which are tylostyles. These 150-320 $\mu \mathrm{m}$ long spicules show a globular swelling at one end and a pointed tip at the other end. The terminal spherical/elliptical knobs have diameters of 6.5$7.3 \mu \mathrm{m}$ in the longitudinal direction and 8.5-9.2 $\mu \mathrm{m}$ in the perpendicular direction; the monaxonial rods are 6.1-6.3 $\mu \mathrm{m}$ thick (Figure 3(d)). Most of the spicules exhibit their spherical ends through the surface (Figure 3(b) and (c)). It is tempting to assume that these globular siliceous balls act as convex lenses that focus the light. The red colored compounds in the sponge tissue have been identified as carotenoids with spectrum maxima at 400 and $430 \mathrm{~nm}$ (Figure $3(\mathrm{e})$ and (f)). The major components present in the sponge 

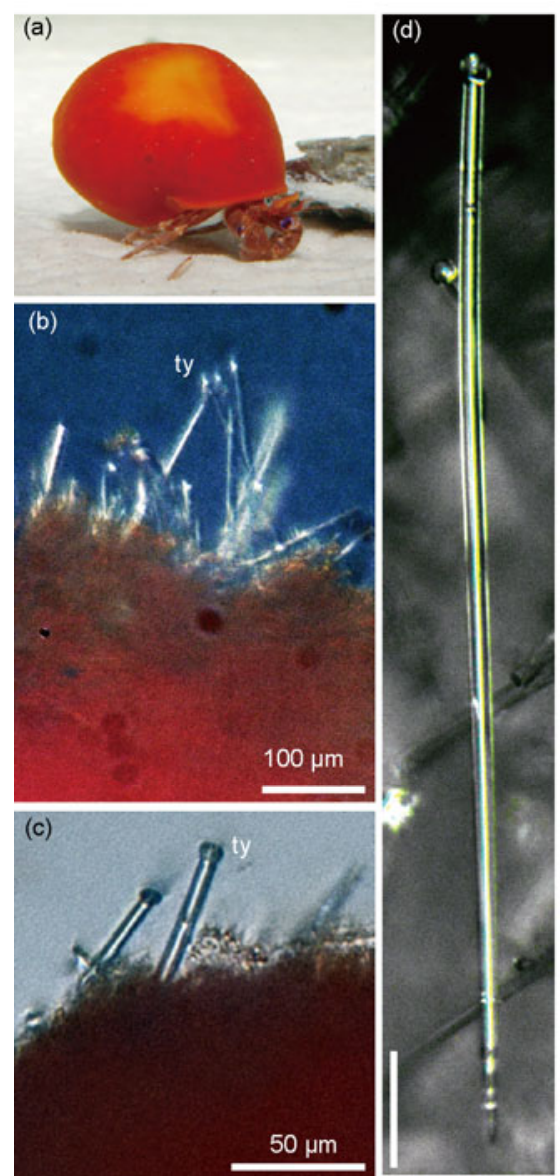
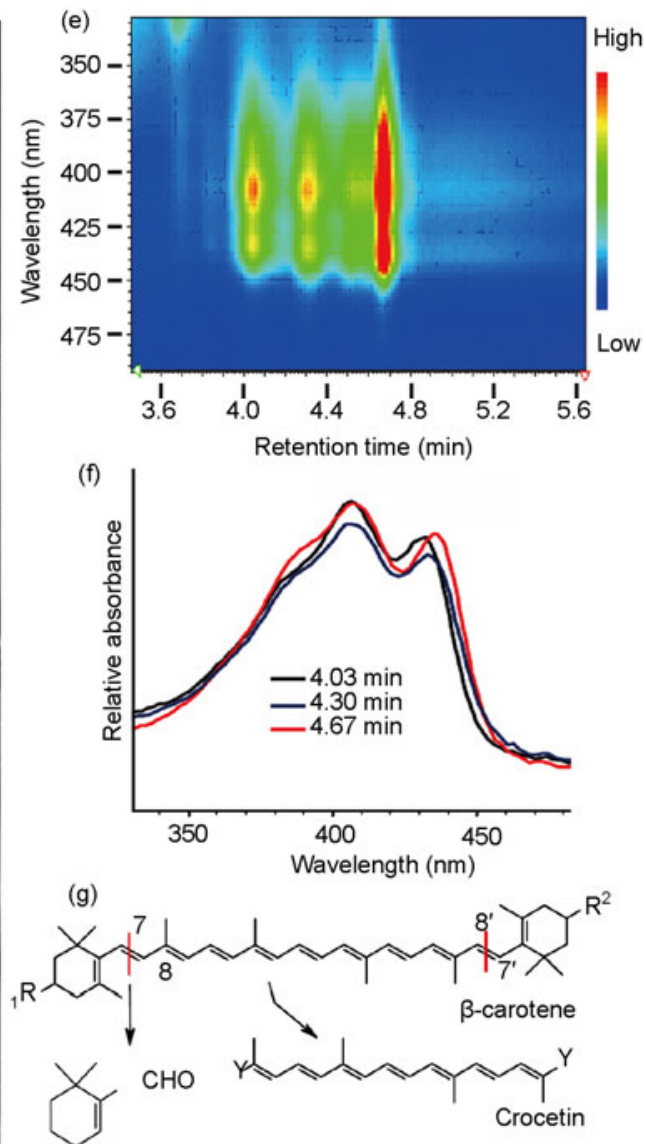

Figure 3 Light harvesting system in S. domuncula. (a) A specimen of the demosponge $S$. domuncula living in the aquarium. (b) and (c) The surface of those sponges show protruding spicules, tylostyles (ty), which expose their spherical/elliptical knobs to the external environment, while the tips remain inside. (d) An isolated tylostyle, with one spheroidal ball-like terminus and a spine at the other terminus. (e) Extraction of the pigments from the tissue of $S$. domuncula. The extracts were then shaken out in an organic solvent. The compounds in the organic phase were separated by reversed-phase HPLC. Fractions eluting at a retention time of 4.92, 4.30 and 4.67 min were collected and separated by TLC using the solvent $\mathrm{n}$-hexane: ethanol (99.5:0.5). The spots were visualized with an integrating detector at 475 to $350 \mathrm{~nm}$. (f) The spectrum of the three fractions analyzed in (e). (g) The carotenoids found in $S$. domuncula; they are the degradation products from $\beta$-carotene and have been identified as members of crocetin and $\beta$-cyclocitral. Figure $3 \mathrm{~d}$ was modified from Ref. [25].

are the degradation products from $\beta$-carotene, compounds belonging to the crocetins, red/yellow colored substances, and the volatile $\beta$-cyclocitral (Figure 3(g)).

Based on these data we propose that the $S$. domuncula specimens can collect the dim light within their spherical/elliptical knobs and guide it through the spicules into the interior of the animals. The red pigments, the carotenoids, are - besides of being involved in intermediary metabolism -assumed to act as a sensitizer and as a dopant for the silica light waveguide.

\section{Light generating machinery: luciferase- luciferin}

Intensive research on bioluminescence started with the elucidation of the luciferin-luciferase reaction in the firefly
Photinus pyralis [22]. Later, molecular biology studies revealed that luciferase is a generic name and includes a series of different classes of luciferase molecules. Luciferin has been identified in (i) bacteria as reduced riboflavin phosphate, $\mathrm{FMNH}_{2}$, that undergoes oxidation by luciferase in association with long chain aldehyde and an oxygen molecules; (ii) dinoflagellates where it is conformationally shielded from luciferase at the basic $\mathrm{pH}$ of 8 , but is released and accessible to oxidation at the more acidic $\mathrm{pH}$ of 6; (iii) the marine ostracod Vargula which acquires it by ingestion of bacteria; (iv) coelenterates where its activity is controlled by the concentration of $\mathrm{Ca}^{2+}$ and the molecule shares homology with the calcium-binding protein calmodulin; (v) insects (fireflies, glowworms and click beetles) as luciferin, a benzothiazole, that in fireflies (Photinus or Luciola) has the unique property of requiring ATP as a co-factor to convert into an active molecule $[23,24]$. 

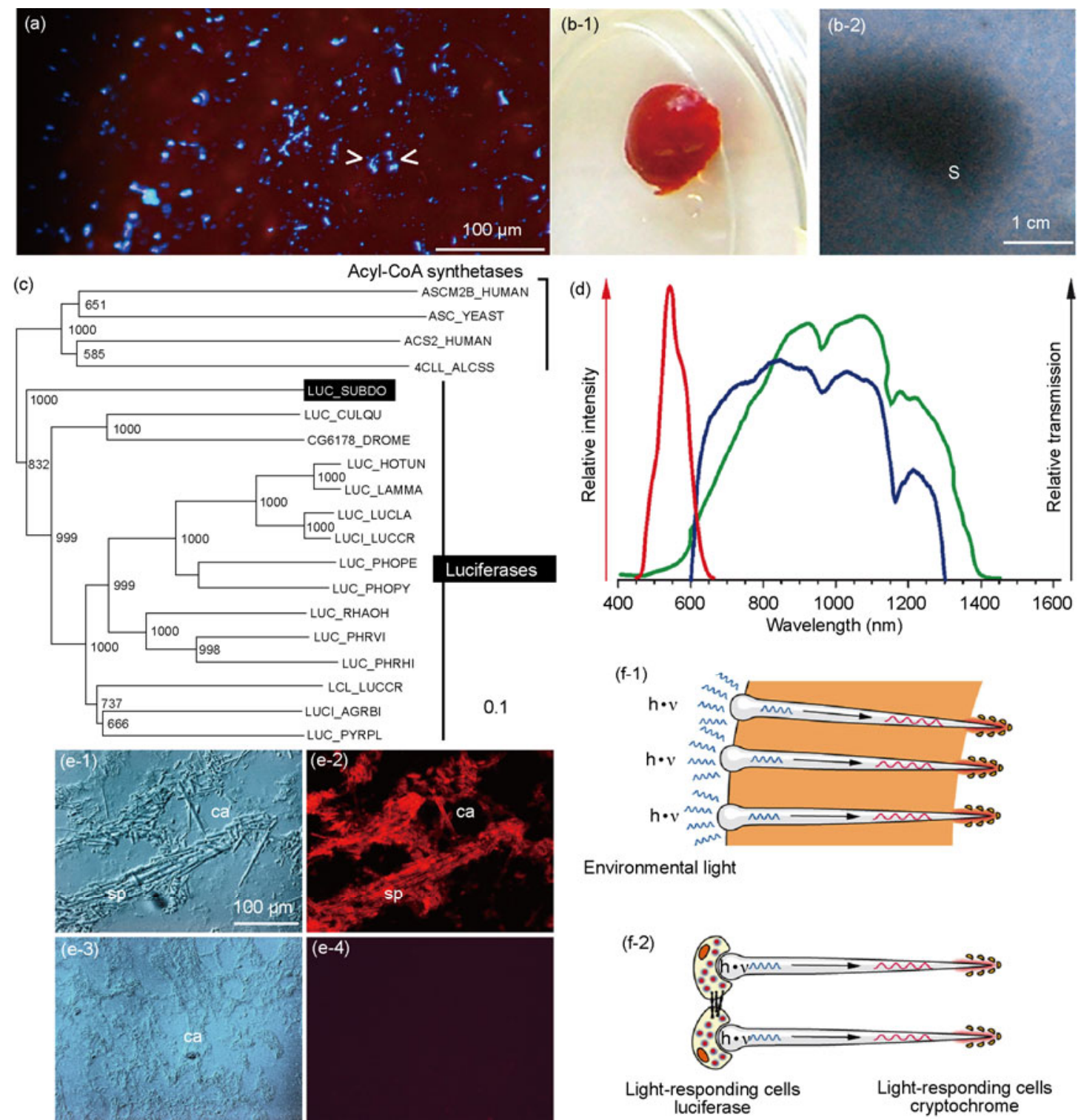

Environmental light

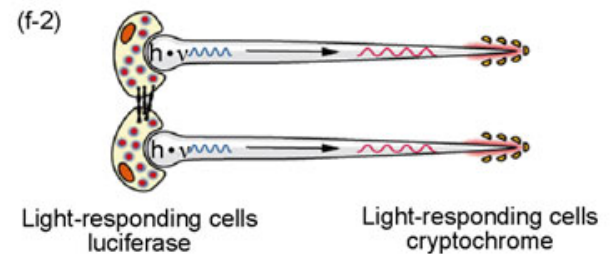

Figure 4 Light generating system in S. domuncula: luciferase. (a) A tissue slice is exposed to blue light. This light is transmitted through the tissue and flashes $(><$ ). (b) Generation of light (bioluminescent flashing) by the tissue from $S$. domuncula (b-1) was detected by a chemiluminescent detection film (b-2). Cubes of a dark adapted animal were exposed to the detection film. A dark spot (s) on the film could be resolved following development with X-ray films. (c) Phylogenetic analysis of $S$. domuncula luciferase (LUC_SUBDO; accession number FM201300). The deduced protein sequence was aligned with the related luciferase from $P$. pyralis (LUC_PHOPY) and the human acyl-CoA synthetase family member 2 (ACS2_HUMAN) as well as the following related luciferases from the fireflies Hotaria unmunsana (LUC_HOTUN), Luciola lateralis (LUC_LUCLA), Lampyroidea maculata (LUC_LAMMA), Photuris pennsylvanica (LUC_PHOPE), Rhagophthalmus ohbai (LUC_RHAOH), the luciferin 4-monooxygenase from the Japanese firefly Luciola cruciata (LUCI_LUCCR), the beetle Pyrophorus plagiophthalamus (LUC_PYRPL), the railroad-worms Phrixothrix vivianii (LUC_PHRVI) and Phrixothrix hirtus (red-bioluminescence eliciting luciferase) (LUC_PHRHI), the mosquito Culex quinquefasciatus (luciferin 4-monooxygenase) (LUC_CULQU), the luciferase homologue from Agrypnus binodulus (LUCI_AGRBI), the paralogous genes of the Japanese firefly Luciola cruciata (LCL_LUCCR) as well as the hypothetical gene from Drosophila melanogaster (CG6178_DROME). In addition, members of the human acyl-CoA synthetase medium-chain family member 2B (CSM2B_HUMAN) and the acetyl CoA synthetase of Saccharomyces cerevisiae (ACS_YEAST) have been included. The tree was calculated and rooted with the plant sequence from A. thaliana as the outgroup. The scale bar indicates an evolutionary distance of 0.1 aa substitutions per position in the sequence. (d) Superposition of the relative light transmission curves between 400 and $1600 \mathrm{~nm}$ through one Hyalonema sieboldi basal spicule (blue) and one Monorhaphis chuni GBS (green), and the bioluminescence emission spectrum of the $S$. domuncula luciferase at $\mathrm{pH} 8.0$ (red). (e) Localization of luciferase in tissue from $S$. domuncula by immunofluorescence staining. Tissue slices that were exposed to light were prepared and reacted with antibodies (Po$\mathrm{Ab}-\mathrm{aLUCSUBDO}$ ) that have been raised against recombinant luciferase. (e-1) The tissue section was inspected with Nomarsky interference contrast optics. The canals (ca) and the spicules (sp) can be distinguished within the tissue. (e-2) A section that was reacted with PoAb-aLUCSUBDO and subsequently with Cy3-conjugated IgG. (e-3) In a control series, a section was inspected with interference optics or (e-4) had been incubated with preimmune serum and then with Cy3-conjugated IgG. (f) Proposed photoreception/phototransduction system of sponges. (f-1) The combination of a cryptochrome and spicules arranged at the surface of the sponge act as a sensory system for environmental light. (f-2) Concurrently, biogeneous light generated within the interior of the animals by luciferase may also be transduced/detected by the same system. (b) and (c) were modified from Ref. [25]. (e) and (f) were modified from Ref. [29]. 
Light transmission through spicules can be demonstrated not only on isolated spicules but also after exposure of the tissue slices to blue light (Figure 4(a)). The first evidence that $S$. domuncula has the ability to flash came from an exposure study with tissue from a dark adapted animal. If a tissue sample (Figure 4(b-1)) was connected to a chemiluminescent detection film for $24 \mathrm{~h}$, a clear signal could be detected following the development with a chemiluminescent substrate (Figure 4(b-2)).

In order to identify the luciferase in $S$. domuncula our EST database was screened with the main focus on the presence of polypeptides comprising the characteristic domains for metazoan luciferase [25], e.g., the acetyl-coenzyme A (acyl-CoA) synthetase region and the luciferinbinding site residues. This database, which comprises 30000 ESTs, can be considered to cover most of the genes in sponges. One fragment was identified that shared high sequence similarity to the firefly luciferase. The firefly luciferase converts the substrate luciferin, a heterocyclic carboxylic acid, and ATP into the corresponding luciferyl adenylate, and under consumption of molecular oxygen to an electronically excited state product that emits a photon of visible light [26]. The binding site for luciferin to luciferase from $P$. pyralis had been studied in detail [26]. Data show that the dehydroluciferin moiety interacts in the hydrophobic pocket of the luciferase from Luciola cruciata with the regions consisting of $\alpha-8$ (amino acid residues), $\beta-12, \beta-13$ $\beta-14, \beta-15$ and an additional loop. During the flashing reaction, luciferin is converted by the monooxygenase luciferase to oxyluciferin [27]. In turn, oxyluciferin displays a strong inhibitory effect on the firefly luciferase reaction in a competitive manner. Hence, intensive screening for luciferin-regenerating system(s) started and resulted in the identification and molecular cloning of a luciferin-regenerating enzyme from $P$. pyralis and related insects. This enzyme converts oxyluciferin in the presence of $D$-cysteine to the intermediate 2-cyano-6-hydroxybenzo-thiazole.

Sequence comparison of the $S$. domuncula luciferase cDNA was performed and the highest sequence similarity was found to be the luciferase protein from $P$. pyralis [25] (Figure 4(c)). Lower similarities to the 4-coumarate-CoA ligases and the acetyl CoA synthetases were observed, with only $36 \%$ similarity ( $19 \%$ identity) to the acetyl CoA synthetase from Saccharomyces cerevisiae.

Following the completion of the molecular biology studies we had unequivocally demonstrated the presence of the gene encoding luciferase [25] and we also reported on the existence of a gene for the luciferin-regenerating enzyme. Furthermore, after demonstrating for the first time both the property of the demosponge spicules to act as optical waveguides and the capacity of the animals to produce light in crude extracts, we showed that the recombinant luciferase was bioactive and caused bioluminescence activity in vitro. Finally we showed that the level of expression of the luciferase gene was strongly down-regulated if cells from $S$. domuncula were constantly exposed to light (Figure 4(e), specifically Figure 4(e-2)). For those studies, primmorphs, a special form of 3D-cell culture [28], were used.

The emission spectrum of the $S$. domuncula luciferase revealed a maximum peak at $548 \mathrm{~nm}$ and a minor peak at $590 \mathrm{~nm}$ (pH 8.0) (Figure 4(d)), concurrent with the bioluminescence spectra of the firefly luciferase/luciferin system that generally vary in the range of 520 to $620 \mathrm{~nm}$. In turn, the bioluminescence emission spectrum, with peaks at 548 and $590 \mathrm{~nm}$, overlaps, as expected, with the steep slope of the curve of the transmission spectra for light through hexactinellid spicules (Figure 4(d)). These results indicate that spicules also act as waveguides for biogenous light in sponges in vivo. Although in sponges the neuronal cell types of Eumetazoa are absent, post-synaptic scaffolds and proneural genes are present and even more interesting is the observation of electric impulse conduction in the hexactinellid Rhabdocalyptus dawsoni [3].

$S$. domuncula spicules are localized in a highly ordered pattern, immediately below the surface cell layers, in palisade-like arrays [29]. Interestingly, the orientation of tylostyles is so that the pointed rods are primarily directed towards the center of the animal, the medulla, whereas the knobs are mainly directed towards the surface (Figure 3(c)). This directionality may imply light guidance from outside into the sponge body (Figure 4(f)). This is supported by the light conditions of the habitat: the animals are found exclusively in shallow waters $(20-30 \mathrm{~m})$. In these coastal waters light within a shorter range of wavelengths (around $500 \mathrm{~nm}$ ) is preferentially transmitted, compared with offshore ocean water. Interestingly, this range of wavelengths corresponds to the bioluminescence emission spectrum of the $S$. domuncula luciferase. Hence, it can be deduced that spicules exposed at the surface of the sponge, absorb/harvest and transmit light to a cryptochrome-containing photoreceptive system, see below.

\section{Flashing sponge: light emission from S. domuncula}

Somehow surprising was the observation that $S$. domuncula can flash light [30]. Immediately after ablation of the cortical, surface cell layers from a dark-adapted sponge were removed and the sponge placed into a beaker with seawater under constant aeration. The beaker was then positioned in a padded dark box with the freshly cut sponge surface as close as possible to the photomultiplier tube (PMT; Figure 5(a)). The PMT was connected in series with a frequency counter and data acquisition unit outside the box. The specimen was fixed in position with forceps. Light (photon) emission was recorded within a range of $300-890 \mathrm{~nm}$. The dark count rate of the detector was $10 / \mathrm{s}$ and, therefore, around one dark count per measuring interval. The quantum 
(a)

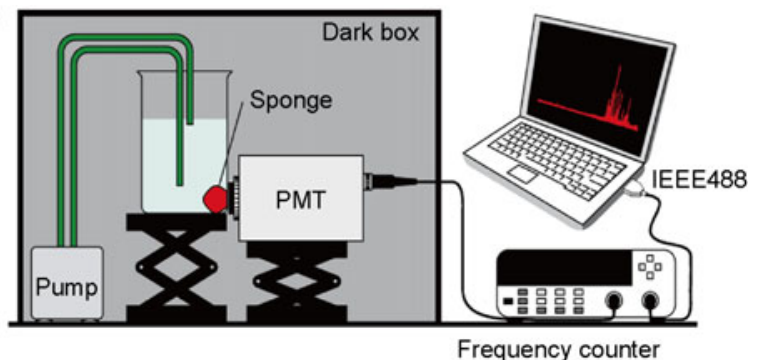

(b)

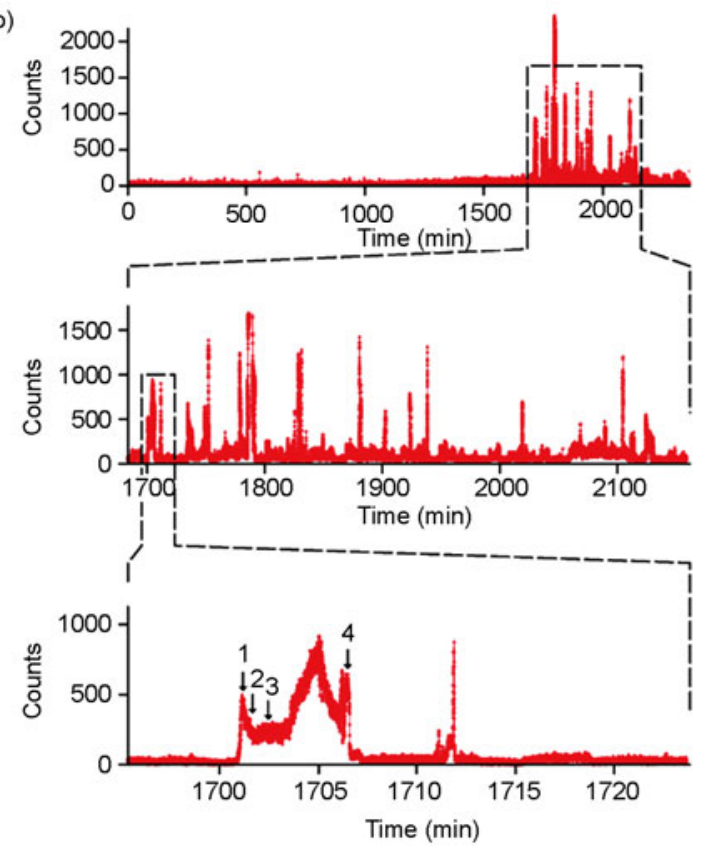

Figure 5 Light flashing by the sponge $S$. domuncula. (a) The photomultiplier tube (PMT) and the sponge specimen were placed in a vibration-free dark box. The sponge was kept under constant water circulation and fixed by means of forceps, facing the PMT. The data acquisition and processing devices were located outside the box and consisted of the frequency counter, connected via IEEE488 interface to a PC. (b) Light generation/flashing by S. domuncula. Typically, flashing started after a lag phase of 1400-2000 min. In the representative experiment shown here the first signals (counts, reflecting the photon intensities) were recorded after a period of $1688 \mathrm{~min}$. The strongest signals were detected until approximately $1900 \mathrm{~min}$ after the start of the experiment. Magnification of the peaks revealed a characteristic shape; one multi-peak flash sequence is shown between 1702 and $1707 \mathrm{~min}$. Such a typical flashing can be divided into an initial strong burst (1), followed by an exponential decrease (2), which then enters a short plateau phase (3) and one flash unit ends with a sudden end (4). Modified from Ref. [30].

efficiency $(\mathrm{QE})$ of the detector was $15 \%$ and within in the range of visible wavelengths. The counting rate was close to $100 \%$. Based on these properties, detection was limited to around 100 emitted photons.

During a typical experiment, the sponge specimens started to emit light after a lag phase of 1400-2000 min $(23-33 \mathrm{~h})$. A characteristic recording profile is shown in Figure 5(b). In this experiment the specimen started to flash after 1688 min of complete inactivity. Almost at the onset of flashing the highest number of counts (2250 counts) was recorded. The activity lasted until ca. $4000 \mathrm{~min}(66 \mathrm{~h})$. This observation was the first indicator that flashing of the sponge specimens correlated with the onset of cortical cell regeneration. Magnification of the recorded flashes revealed a characteristic shape, with an initial and major, perhaps overshooting, burst followed by a (bi)exponential decrease, passing into a plateau phase. At times, this pattern was immediately repeated. Finally, the flashing activity was abruptly terminated, as indicated by a sudden drop in the number of counts detected (Figure 5(b); lowest panel).

These data show that after reconstitution of the cortex and the phase-dependent expression of the building blocks of the proposed photosystem, sponges start to generate light impulses. During the first $28 \mathrm{~h}$ post-ablation of surface tissue, only short, weak, and rare flashes occur. This period of dormancy is terminated by heavy activity. During the subsequent $10 \mathrm{~h}$ of observation, peaks were recorded with up to 2250 counts. Currently, it is only possible to speculate about the function of the proposed light flashing/signaling circuit during reconstitution of the damaged poriferan tissue. In earlier reports, it had been proposed that in corals the light-responsive cryptochrome system is involved in the synchronization of, for example, mass spawning [31], concurrently suggesting that the coral cryptochrome system controls an ancient circadian clock-related rhythm.

\section{Light detection system: the cryptochrome}

The discovery of the cryptochrome(s), which are crucially involved in light harvesting reactions in corals [32] stimulated a systematic screening for homologous poriferan proteins, resulting in the identification of candidate molecules in demosponges ( $S$. domuncula) as well as in hexactinellids (Crateromorpha meyeri) [29]. As in the coral model [32], the expression of poriferan cryptochrome is controlled by light [28]. More specifically, the expression of the $S$. domuncula cryptochrome gene after light exposure (the light source within the spectral range of 330-900 and 700$1100 \mathrm{~nm}$ ) [29] is primarily restricted to the surface zone of the animal, suggesting that the photoactivated protein is compartmentalized in the cortex (the outer tissue layer). Similarly, the expression of poriferan luciferase is lightadapted [25].

The gene expression of cryptochrome is dependent on light. Samples of cell aggregates or tissue were exposed to light for 1-8 h, using a short-pass filter (spectral range, $330-900 \mathrm{~nm}$ ), or long-pass filter (spectral range, 700-1100 $\mathrm{nm})$. The RNA was subsequently extracted. Northern blot analyses then revealed that after dark adaptation of primmorphs or tissues no expression of cryptochrome was detected. However, after $2 \mathrm{~h}$ of light exposure (330-900 $\mathrm{nm})$ an increase in the expression of the cryptochrome was observed, which increased further after 4 or $8 \mathrm{~h}$ of light exposure, both in tissue and in primmorphs. In a crucial set of experiments, quantitative real-time PCR (qPCR) was 
applied to determine cryptochrome transcription over $24 \mathrm{~h}$, including the light-dark transition (Figure 6). Subsequently, cryptochrome expression was correlated to the expression of the housekeeping gene tubulin. Thus, during $12 \mathrm{~h}$ light exposure, cryptochrome expression increased to 0.53 and then decreased, until after $12 \mathrm{~h}$ of darkness a ratio of 0.15 was calculated. Accordingly, cryptochrome expression during light exposure was up to 3.5 -fold higher than in darkness.

\section{The light-response system in sponges}

Taken together, these data demonstrate that exposure of $S$. domuncula (tissue and primmorphs) to light stimulates cryptochrome expression. In contrast to the studies performed with corals [32], the light-induced response in sponges occurs regardless of the spectral range. Since cryptochrome is particularly localized around spicules, it is assumed that skeletal elements operate as waveguides for environmental light that penetrates with significant intensity to water depths of $\sim 20 \mathrm{~m}$ in the northern Adriatic Sea, the natural habitat of $S$. domuncula. Thus, spicules at the surface of the animal may function as collectors that transmit light to the cryptochrome, the putative photosensory receptor of sponges (Figure 7(a)). Concurrently, light produced intracellularly (luciferase-mediated) may be collected and transmitted via the same optical system. In both cases, the cryptochrome is the light photoreceptor. It remains to be studied whether flavin or another chromophore is associated

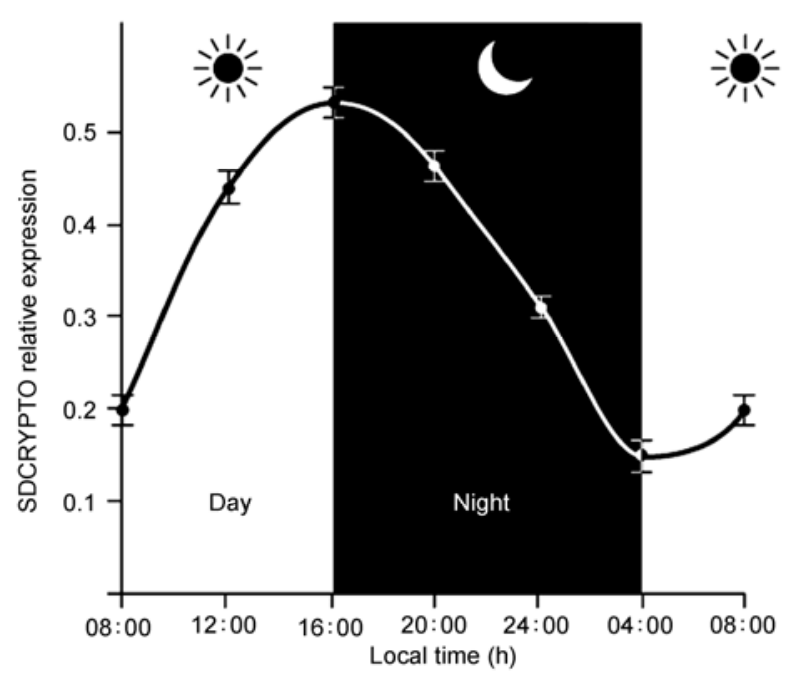

Figure 6 Cryptochrome expression analyses of $S$. domuncula tissue. Dark-adapted samples were exposed to light $(330-900 \mathrm{~nm})$ for $8 \mathrm{~h}(08: 00$ to $16: 00)$ and then kept in darkness for $12 \mathrm{~h}$ (16:00 to 04:00). Following RNA isolation, expression levels of cryptochrome and tubulin (housekeeping gene) were determined through quantitative real-time PCR, and subsequently correlated to determine relative expression levels. Modified from Ref. [29]. with the cryptochrome, for example, avarol/avarone is frequently found in demosponges [33].

\section{Light-dependent alterations of the induction of genes involved in the light-response system}

The findings that in response to light both luciferase and cryptochrome are expressed to a higher level prompted us to search for upstream localized transcription factors (TF) controlling the expression of cryptochrome and luciferase, the key molecules that are involved in the proposed photoreception/photogeneration system in sponges [30]. No poriferan homolog of Pax-6, a molecule that is required for morphogenesis of the visual system in triploblasts [34], could be detected. Therefore, we screened for SOX genes, encoding a family of metazoan-specific TF [35] that play important roles in a variety of developmental processes, particularly during organogenesis [36]. All SOX proteins comprise a highly conserved high mobility group (HMG) box. Of the $>30$ SOX family members known, SOX-2 [37] has been implicated in the early human eye and brain development. In our studies [30] we analyzed the spatiotemporal expression of the $S$. domuncula genes coding for a SOX-related protein, a luciferase, and a cryptochrome, following ablation of the surface cell layers. Shortly after the expression of the potential photosensory proteins (ca. $10 \mathrm{~h}$ ) the specimens emitted light. With this study we for the first time provided data that demonstrates the existence of a genetically controlled light signaling circuit in sponges (Figure 7(b)).

\section{Conclusions}

After acceptance of the new view of the monophyletic origin of all metazoans, including Porifera, and the findings that both the immune system and the body plan are composed of common basic elements in all animals, it was not too surprising to discovery that the ancestral homeobox genes are also present in sponges [3]. The developmental processes controlling the formation of a body axis require a head center; in bilaterians, for example, the Spemann's organizer. The genes that are involved in the establishment of the head organizer during embryogenesis have been grouped into three classes of homeobox genes: (i) the paired-class; (ii) the Antennapedia-class; and (iii) the LIMclass genes. In this area, rapid progress has been made in sponges in the last few years. A paired-class (Pax-2/5/8)gene has been isolated from the freshwater sponge Ephydatia fluviatilis, which encodes a complete, although substantially degenerate homeodomain. In $S$. domuncula, a cDNA encoding an LIM/homeobox protein has been isolated, which comprises high sequence similarity to the related LIM/homeodomain proteins in other animals. 
(a)
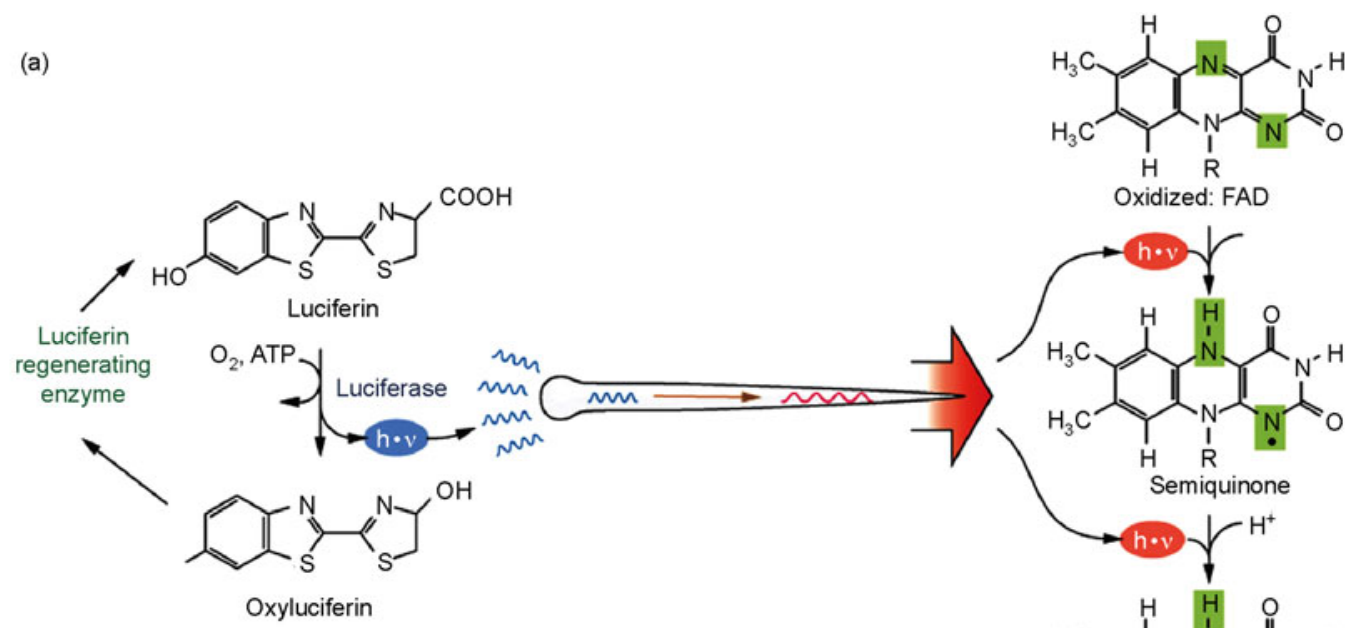

Oxidized: FAD

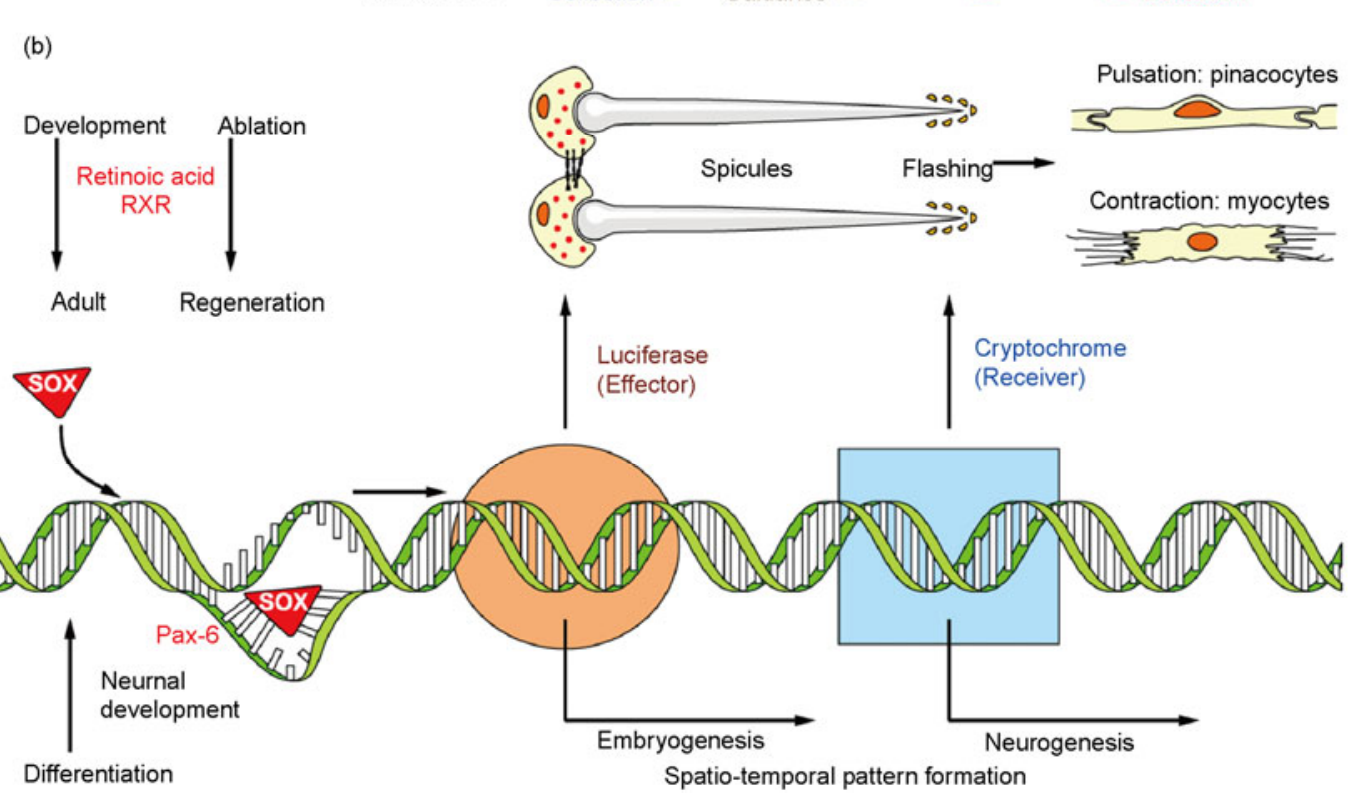

Figure 7 Light transmission circuit in sponges. (a) Schematic detail of this process with bioluminescence as the light source. Luciferase-mediated oxidation of luciferin results in the generation of bioluminescence. Photons are transmitted through spicules and then detected by a chromophore/redox system associated with the cryptochrome. Concomitantly, oxyluciferin is the substrate for the luciferin-regenerating enzyme. (b) Proposed light-flashing signaling circuit. During development and regeneration of sponge tissue, binding of the morphogen retinoic acid to its receptor RXR induces expression of the transcription factor, the SOX-related protein (SOX). In vertebrates, SOX operates during differentiation in general and neuronal development in particular. Flanked by Pax-6, a TF without an apparent poriferan homolog, SOX-2 causes coordinated development of cells during embryogenesis and neurogenesis. In sponges the SOX-related protein causes consecutive expression of luciferase (effector) and cryptochrome (receiver). Both are components of the poriferan light generating/harvesting system, also including siliceous spicules as light waveguides. In this manner, coordinated transmitted signals may regulate, for example, pulsation of pinacocytes or the contraction of myocytes. Modified from ref. [29].

We can now outline that sponges react to light signals with the expression of a light harvesting, transmitting and transforming system (Figure 8). We assume that this circuit explains the coordinate reactions observed in sponges. It appears to be evident that this novel view of the photoresponsive properties of these animals, with the siliceous spicules as light transmitters, will reveal new concepts in the photoperception of animals. 


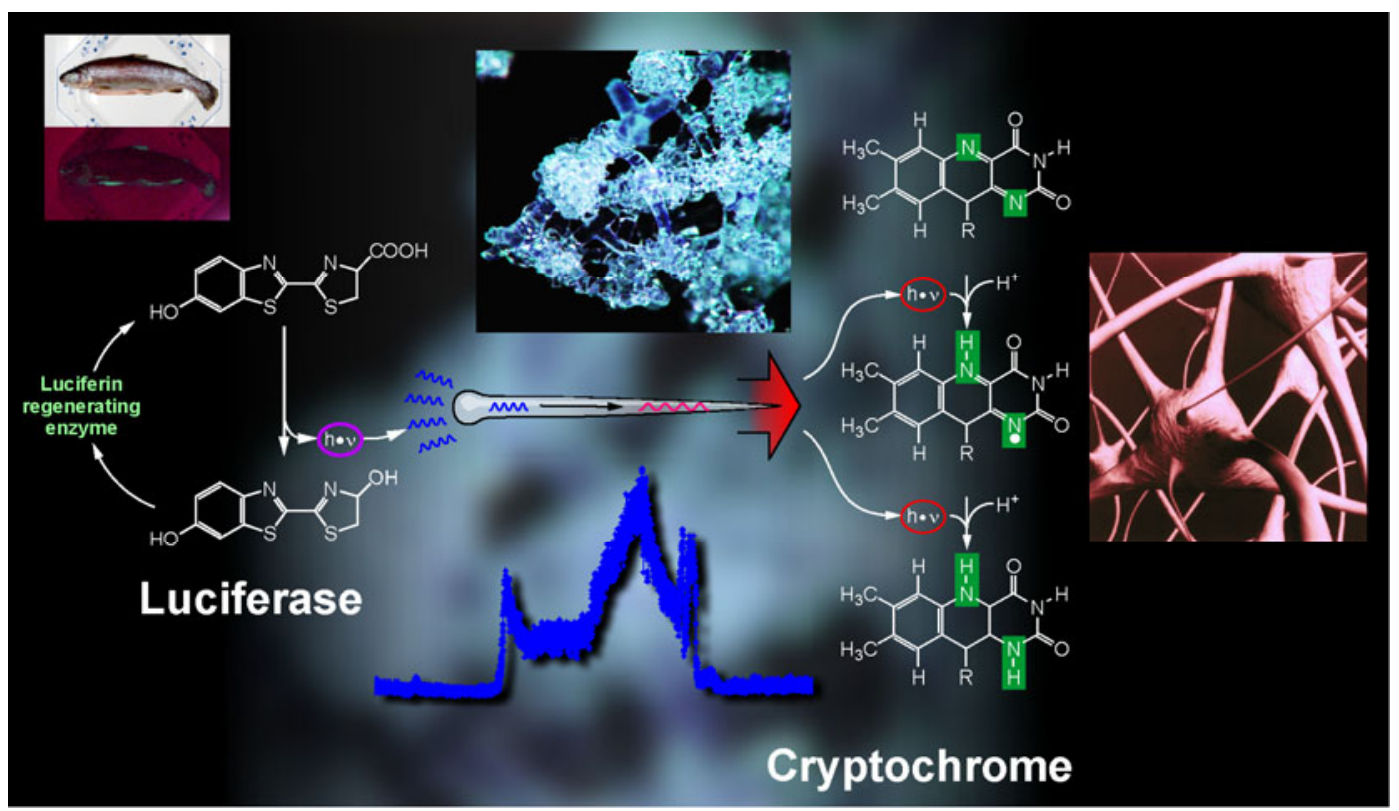

Figure 8 Proposed photoreception system in sponges. Solid evidence is available that light is generated by bioluminescence (luciferase system), which is bundled by silica-based fibers. In contrast, the electrical impulses in higher Metazoa are generated by protein-based reactions. In sponges the photons are recognized by a conformational change of the cryptochrome. We attribute this light sensory system/circuit as a substitute of the neuronal reception and transmission system.

W.E.G.M. is holder of an ERC Individual Advanced Grant $(268476$ BIOSILICA). This work was supported by the German Bundesministerium für Bildung und Forschung (project "Center of Excellence BIOTECmarin"), the Deutsche Forschungsgemeinschaft (Schr 277/10-1), the European Commission/EUREKA (EUROSTARS, 4289-SILIBACTS), the International Human Frontier Science Program, the European Commission (244967-Mem-S, Biomintec 215507, Coreshell 286059, IRSES 246987, SPECIAL 266033, and BlueGenics 311848 Projects), the Public Welfare Project of Ministry of Land and Resources of China (201011005-06) and the International S \& T Cooperation Program of China (2008DFA00980).

1 Müller W E G, Li J, Schröder H C, et al. The unique skeleton of siliceous sponges (Porifera; Hexactinellida and Demospongiae) that evolved first from the Urmetazoa during the Proterozoic: A review. Biogeosciences, 2007, 4: 219-232

2 Wang X H, Hu S, Gan L, et al. Sponges (Porifera) as living metazoan witnesses from the Neoproterozoic: Biomineralization and the concept of their evolutionary success. Terra Nova, 2010, 22: 1-11

3 Müller W E G, Wiens M, Adell T, et al. The Bauplan of the Urmetazoa: The basis of the genetic complexity of Metazoa using the siliceous sponges [Porifera] as living fossils. Int Rev Cytol, 2004, 235: 53-92

4 Müller W E G, Wang X H, Cui F Z, et al. Sponge spicules as blueprints for the biofabrication of inorganic-organic composites and biomaterials. Appl Microbiol Biotechnol, 2009, 83: 397-413

5 Wang X H, Schröder H C, Müller W E G. Giant siliceous spicules from the deep-sea glass sponge Monorhaphis chuni: Morphology, biochemistry and molecular biology. Int Rev Cell Mol Biol, 2009, 273: 69-115

6 Morse D E. Silicon biotechnology: Harnessing biological silica production to construct new materials. Trends Biotechnol, 1999, 17: 230-232

7 Müller W E G. Silicon Biomineralization: Biology-Biochemistry-Molecular Biology-Biotechnology. Berlin: Springer Press, 2003

8 Krasko A, Batel R, Schröder H C, et al. Expression of silicatein and collagen genes in the marine sponge Suberites domuncula is controlled by silicate and myotrophin. Europ J Biochem, 2000, 267:
4878-4887

9 Wiens M, Belikov S I, Kaluzhnaya O V, et al. Axial (apical-basal) expression of pro-apoptotic and pro-survival genes in the Lake Baikal demosponge Lubomirskia baicalensis. DNA Cell Biol, 2006, 25: $152-164$

10 Müller W E G, Wendt K, Geppert C, et al. Novel photoreception system in sponges? Unique transmission properties of the stalk spicules from the hexactinellid Hyalonema sieboldi. Biosens Bioelectron, 2006, 21: 1149-1155

11 Wang X H, Li J, Qiao L, et al. Structure and characteristics of giant spicules of the deep sea hexactinellid sponges of the genus Monorhaphis (Hexactinellida: Amphidiscosida: Monorhaphididae). Acta Zool Sin, 2007, 53: 557-569

12 Harvey E N. Studies on bioluminescence. XIII. Luminescence in the coelenterates. Biol Bull, 1921, 41: 280-284

13 Belas R, Mileham A, Cohn D, et al. Bacterial bioluminescence: Isolation and expression of the luciferase genes from Vibrio harveyi. Science, 1982, 218: 791-793

14 Hervant F, Mathieu J, Barre H. Comparative study on the metabolic responses of subterranean and surface-dwelling amphipods to longterm starvation and subsequent refeeding. J Exp Biol, 1999, 202: 3587-3595

15 Alieva N O, Konzen K A, Field S F, et al. Diversity and evolution of coral fluorescent proteins. PLoS One, 2008, 3: e2680

16 Wiens M, Grebenjuk V A, Schröder H C, et al. Identification and isolation of a retrotransposon from the Lubomirskia baicalensis: Implication in rapid evolution of endemic sponges. Prog Mol Subcell Biol, 2009, 47: 207-234

17 Müller W E G, Belikov S I, Kaluzhnaya O V, et al. Cold stress defense in the freshwater sponge Lubomirskia baicalensis: Role of okadaic acid produced by symbiotic dinoflagellates. FEBS J, 2006, 274: 23-36

18 Wilson T, Hastings J W. Bioluminescence. Annu Rev Cell Dev Biol, 1998, 14: 197-230

19 Ridley S O, Dendy A. Report on the Monaxonida collected by H.M.S 'Challenger'. Report on the Scientific Results of the Voyage of the H.M.S. Challenger During the Years 1873-76. Vol. 20. London: Eyre \& Spottiswoode, 1887

20 Leys S P, Mackie G O, Reiswig H M. The biology of glass sponges. 
Adv Mar Biol, 2007, 52: 1-145

21 Müller W E G, Wang X H, Burghard Z, et al. Bio-sintering processes in hexactinellid sponges: Fusion of bio-silica in giant basal spicules from Monorhaphis chuni. J Struct Biol, 2009, 168: 548-561

22 Strehler B L, McElroy W D. Purification of firefly luciferin. J Cell Physiol, 1949, 34: 457-466

23 Meighen E A. Molecular biology of bacterial bioluminescence. Microbiol Rev, 1991, 55: 123-142

24 Greer L F, Szalay A A. Imaging of light emission from the expression of luciferase in living cells and organisms: A review. Luminescence, 2002, 17: 43-74

25 Müller W E G, Kasueske M, Wang X H, et al. Luciferase a light source for the silica-based optical waveguides (spicules) in the demosponge Suberites domuncula. Cell Mol Life Sci, 2009, 66: 537-552

26 Branchini B R, Ablamsky D M, Murtiashaw M H, et al. Thermostable red and green light-producing firefly luciferase mutants for bioluminescent reporter applications. Anal Biochem, 2007, 361: 253-262

27 Gomi K, Kajiyama N. Oxyluciferin, a luminescence product of firefly luciferase, is enzymatically regenerated into luciferin. J Biol Chem, 2001, 276: 36508-36513

28 Müller W E G, Wiens M, Batel R, et al. Establishment of a primary cell culture from a sponge: Primmorphs from Suberites domuncula. Marine Ecol Prog Ser, 1999, 178: 205-219

29 Müller W E G, Wang X, Schröder H C, et al. A cryptochrome-based photosensory system in the siliceous sponge Suberites domuncula
(Demospongiae). FEBS J, 2010, 277: 1182-1201

30 Wiens M, Wang X, Unger A, et al. Flashing light signaling circuit in sponges: endogenous light generation after tissue ablation in Suberites domuncula. J Cell Biochem, 2010, 111: 1377-1389

31 Levy O, Dubinsky Z, Achituv Y. Photobehavior of stony corals: Responses to light spectra and intensity. J Exp Biol, 2003, 206: 40414049

32 Levy O, Appelbaum L, Leggat W, et al. Light-responsive cryptochromes from a simple multicellular animal, the coral Acropora millepora. Science, 2007, 318: 467-470

33 Sarma A S, Daum T, Müller W E G. Secondary metabolites from marine sponges: Part I: Origin and chemistry of new metabolites, and synthetic studies; Part II: Biological properties of new metabolites and physiological activities of avarol and related compounds isolated from Dysidea sp. Akademie gemeinnütziger Wissenschaften zu Erfurt. Berlin: Ullstein-Mosby Verlag, 1993. 1-168

34 Gehring W J, Ikeo K. Pax 6: Mastering eye morphogenesis and eye evolution. Trends Genet, 2009, 15: 371-377

35 Koopman P, Schepers G, Brenner S, et al. Origin and diversity of the Sox transcription factor gene family: Genome-wide analysis in Fugu rubripes. Gene, 2004, 328: 177-186

36 Wegner M. From head to toes: The multiple facets of SOX proteins. Nucleic Acids Res, 1999, 27: 1409-1420

37 Hagstrom S A, Pauer G J, Reid J, et al. SOX2 mutation causes anophthalmia, hearing loss, and brain anomalies. Am J Med Genet, 2005, 138: 95-98

Open Access This article is distributed under the terms of the Creative Commons Attribution License which permits any use, distribution, and reproduction in any medium, provided the original author(s) and source are credited. 